\title{
Ki67 Proliferative Index and Peritumoral Brain Edema in Meningiomas: Do They Correlate? A Clinical Study on 56 Patients
}

\author{
Nour Imam1 ${ }^{1}$, Ahmed I. Elghriany' ${ }^{1}$, Ahmed M. Elshanawany1, Ahmed A. S. Elhakeem² \\ ${ }^{1}$ Department of Neurosurgery, Faculty of Medicine, Assiut University, Assiut, Egypt \\ ${ }^{2}$ Department of Pathology, Faculty of Medicine, Al-Azhar University, Assiut Branch, Assiut, Egypt \\ Email: nourimam@med.au.edu.eg
}

How to cite this paper: Imam, N., Elghriany, A.I., Elshanawany, A.M. and Elhakeem, A.A.S. (2019) Ki67 Proliferative Index and Peritumoral Brain Edema in Meningiomas: Do They Correlate? A Clinical Study on 56 Patients. Open Journal of Modern Neurosurgery, 9, 461-471.

https://doi.org/10.4236/ojmn.2019.94045

Received: September 12, 2019

Accepted: October 28, 2019

Published: October 31, 2019

Copyright $\odot 2019$ by author(s) and Scientific Research Publishing Inc. This work is licensed under the Creative Commons Attribution International License (CC BY 4.0).

http://creativecommons.org/licenses/by/4.0/

(c) $\underset{\mathrm{EY}}{\mathrm{C}}$ Open Access

\begin{abstract}
Introduction: Meningiomas are the most common type of extra-axial neoplasm. Peritumoral brain edema (PTBE) can be seen around meningiomas while it may be absent in others. Despite that Ki67 proliferative index has been previously correlated with meningioma grades, no definite relationship has been established in relation to PTBE in meningioma patients. Objective: Correlate the peritumoral brain edema with the Ki67 proliferative index of meningiomas. Patients \& Methods: Aclinical prospective study was conducted on 56 patients ( 47 women, 9 men; mean age $50.89 \pm 12.55$ years) diagnosed with meningiomas. All patients were evaluated regarding the presence of brain edema surrounding the lesion in pre-operative neuroimaging using T2W and FLAIR MR images. Immunohistochemical staining of Ki67 index (representing proliferative activity) was done. Correlation between presence of PTBE and Ki67 index values was evaluated. Results: PTBE was found in nearly half of the patients (48.2\%), while the remaining $(51.8 \%)$ of patients did not exhibit PTBE in their pre-operative neuroimaging. The mean value of Ki67 index in meningioma patients with PTBE was $4.83 \%$ compared to a value of $1.83 \%$ in patients without PTBE, $\mathrm{P}$ value $=0.014$. Conclusion: High Ki67 indices are evident in meningiomas with surrounding peritumoral brain edema (PTBE).
\end{abstract}

\section{Keywords}

Meningioma, Ki67, Proliferative Index, Peritumoral, Brain Edema

\section{Introduction}

Meningiomas are the most common primary non-glial intracranial tumours [1]. 
The 2016 World Health Organization (WHO) classification states 15 distinct meningioma variants grouped into three grades, WHO grade I (benign), grade II (intermediate) and grade III (malignant) [2]. Brain imaging with contrast-enhanced CT or MR imaging is the most common method of diagnosing, monitoring, and evaluating response to treatment [3].

Meningiomas tend to be benign, and may remain clinically silent. However, they could lead to significant morbidity and mortality, that is not only be caused by the size and location of the tumor but also due to the presence of peritumoral brain edema (PTBE). PTBE increases the mass effect and causes more elevated intracranial pressure, and as a consequence it may lead to eventual cerebral herniation. It is also considered a prognostic factor for the complexity of surgical resection and may affect surgical outcome [4]. PTBE is best visualized on T2W or FLAIR MRI images, and correlates with size, rapid growth, location and invasion in the case of malignant meningiomas. The underlying mechanisms for PTBE may relate to venous stasis, thrombosis, compressive ischaemia and aggressive growth [1]. Various degrees and shapes of edema have been reported. The extent of edema may vary considerably ranging from absent or mild edema to nearly 2 - 3 times the size of the tumor. It is a well-known fact that also small meningiomas may cause surprisingly pronounced PTBE [5], which is why the question was raised if factors different from the mere tumor location and tumor size may contribute to the occurrence and extent of PTBE [6] [7] [8].

$\mathrm{Ki} 67$ index is one of the most reliable markers of cell proliferation, yet there is little data about the relationship between Ki67 index and peritumoral edema in meningiomas. As PTBE is a common finding in meningiomas, and Ki67 indices are recently becoming more and more incorporated in meningioma assessment, we aimed to clarify whether a relationship between the two could be identified.

\section{Patients and Methods}

This is a clinical prospective study conducted on 56 patients diagnosed with meningiomas, whom were surgically treated at the Department of Neurosurgery, Assiut University Hospital during the period between January 2016 to May 2018. Patients were followed up until January 2019. Neuroimaging studies; mainly CT and MRI scans of the patients were performed pre-operatively. Inclusion Criteria for this study included patients with intracranial meningiomas that were diagnosed through pre-operative neuroimaging and post-operative histopathological examination and whom were fit for surgery. Exclusion criteria were patients unfit for surgery, and those with other additional associated lesions.

Tumor volume was assessed on contrast enhanced T1W MRI, while edemavolume was measured on T2W and FLAIR MRI, Edema Index (EI) was calculated by the formula $\mathrm{EI}=(V$ tumor + edema $) /(V$ tumor $)$. If there is no edema, EI $=1$. The volume of the tumor and extent of peritumoral edema were measured from the MRI where the longest horizontal diameters of the tumor were measured from the axial image. The vertical diameter of the tumor was measured from the coronal image. The three values were multiplied to measure the volume 
of the lesion. PTBE volume was measured in a similar manner by measuring the high signal intensity region in T2WI/FLAIR that was clearly distinguished from normal tissues, including the tumor (Figure 1).

All included meningiomas were surgically resected at the Neurosurgery Department, Assiut University Hospital. Post-operative histopathological and immunohistochemical examination was performed by the same specialized neuropathologist to eliminate interlaboratory variability. Histopathological examination was done on haematoxylin and eosin stained sections and immunohistochemical examination of Ki67 proliferative index is performed on poly-L-Lysine coated slides, evaluation was performed under high power objective (magnification $400 \times$ ), distinct brown nuclear staining is recorded as positive. The areas with the highest number of positive tumor nuclei (hot spots) were selected and an average of 1000 tumor cells from the areas of maximum labeling were counted for analysis [9] (Figure 2).
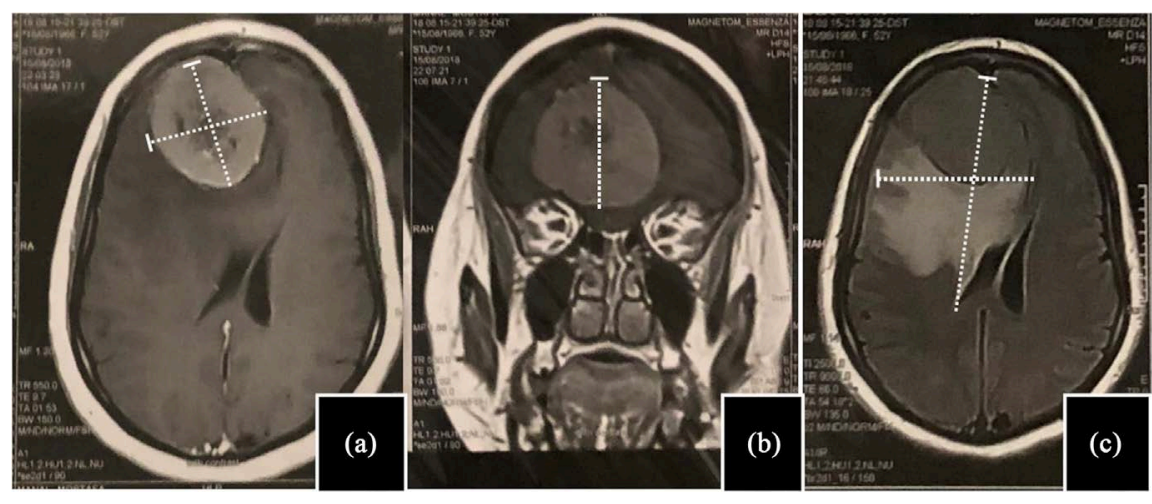

Figure 1. (a) Axial MRI T1WI Gd+ demonstrating a right frontal meningioma, measurement of the longest diameter and vertical diameter is done to assess tumor volume; (b) Coronal image of the same lesion to measure the axial diameter; (c) MRIFlair sequence to better assess the peritumoral edema shown surrounding the lesion as a hyper intense irregular signal, measurement of the edema volume is done in the same manner.

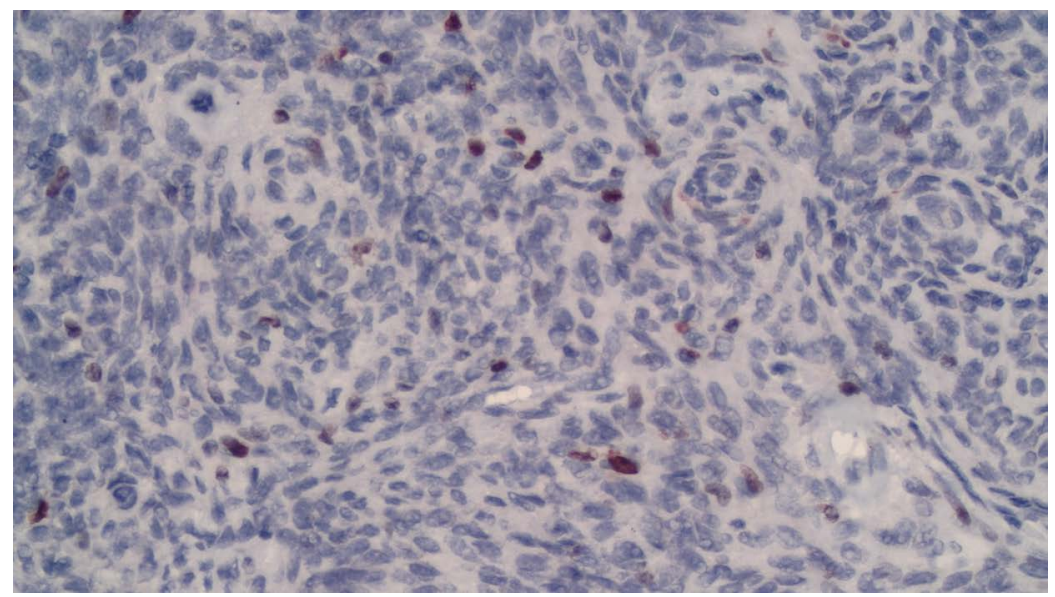

Figure 2. Immunohistochemical expression of Ki67 showing nuclear staining of Ki67 positive cells of meningioma seen in dark red/brown color, under high power microscope (magnification $\times 100$ ). 
Statistical analysis for different variables was done using IBM SPSS Statistics, version 20. Correlation between parametric data was done with $\mathrm{t}$-test and non parametric data with $\chi^{2}$-test. $\mathrm{P}$ value of 0.05 was estimated as the cut off for significance.

\section{Results}

\section{Demographic Data}

This study included 56 cases of intracranial meningiomas that were surgically treated. Age of patients ranged between 17 - 75 years with a mean age of 50.89 years, female gender was predominant among the patients represented by 47 female patients (83.9\%) while 9 patients were males (16.1\%). The most common locations for meningiomas were convexity and parasaggital (Table 1).

\section{Tumor Volume, PTBE and KI67 Index}

The mean tumor volume was $38.62 \mathrm{~cm}^{3}$, PTBE was found in $48.2 \%$ of the study group while absent in $51.8 \%$ (Table 2).

There was no statistical difference in tumor volumes between male and female genders $(t=-1.486 ; \mathrm{P}=0.191)$ and the mean edema index was 4.27. Ki67 index represents the proliferative activity of meningiomas, it was evaluated in all cases postoperatively, the mean value of Ki67 index in this study was $3.28 \%$, with a minimal and maximal value of $0.3 \%$ and $19.5 \%$ respectively (Table 3 ).

Regarding Ki67 index, the mean value was $3.28 \%$ for all patients in the study so for Ki67 and PTBE correlation analysis, the cases were grouped as either Ki67 index above or below 3\%. 36 patients had Ki67 indices below 3\%; 12 of which had associated PTBE while 24 did not.

There were 20 patients that had Ki67 indices above 3\%; 15 of which (75\%) showed PTBE while 5 patients (25\%) did not show PTBE. The mean value of Ki67 index in all patients with positive PTBE was 4.83 compared to a mean value of 1.83 in those without PTBE (Figure 3). P value was calculated to be 0.014 indicating strong correlation between PTBE and Ki67 index (Table 4).

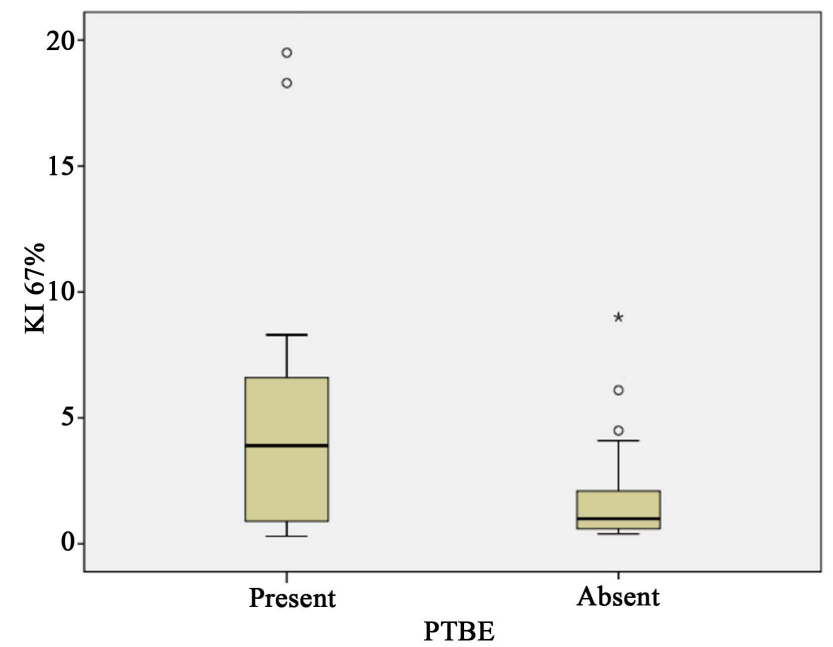

Figure 3. Box plot with scatter diagram showing Ki67 values correlated with PTBE. 
Table 1. Demographic data of patients in the study.

\begin{tabular}{cccc}
\hline Variable & $\boldsymbol{n}(\%)$ & Variable & $\boldsymbol{n}(\%)$ \\
\hline Age & & Location & \\
\hline$<40$ years & $17(14.3 \%)$ & Convexity & 20 \\
$40-49$ years & $14(25 \%)$ & Parasaggital & 17 \\
$50-59$ years & $14(25 \%)$ & Sphenoid Wing & 9 \\
$60-69$ years & $3(5.3 \%)$ & Ollar/Suprasellar & 4 \\
$\geq 70$ years & $50.89 \pm 12.55(17 \%-75 \%)$ & Foramen Magnum & 2 \\
Mean Age \pm SD Range & & Petroclival & 1 \\
Gender & $47(83.9 \%)$ & & \\
Female & $9(16.1 \%)$ & & \\
Male & & & \\
\hline
\end{tabular}

Table 2. PTBE incidence.

\begin{tabular}{ccc}
\hline PTBE & Patients No. (56) & Gender \\
\hline Present (+ve) & $27(48.2 \%)$ & F: 21 M: 6 \\
Absent (-ve) “EI = 1" & $29(51.8 \%)$ & F: 26 M: 3 \\
\hline
\end{tabular}

F: Female, M: Male.

Table 3. Statistical values: Tumor volume, Edema index, Ki67 index.

\begin{tabular}{ccc}
\hline \multicolumn{3}{c}{ Preoperative tumor volume $\left(\mathrm{cm}^{3}\right)$} \\
\hline Number & Mean & Standard Deviation \\
\hline 56 & 38.62 & 31.71 \\
Number & Edema Index (EI) & \\
56 & Mean & Standard Deviation \\
& 4.27 & 3.52 \\
Number & Ki67 Index (\%) & Standard Deviation \\
56 & Mean & 3.96 \\
\hline
\end{tabular}

Table 4. Correlation between Ki67 index and PTBE.

\begin{tabular}{cccc}
\hline Ki67 index & PTBE (-ve) & PTBE (+ve) & Total \\
\hline Ki67 $<3 \%$ & $24(66.67 \%)$ & $12(33.33 \%)$ & $36(100 \%)$ \\
Ki67 $>3 \%$ & $5(25 \%)$ & $15(75 \%)$ & $20(100 \%)$ \\
Total & $29(51.79 \%)$ & $27(48.21 \%)$ & $56(100 \%)$ \\
Total & Mean Ki 67\% PTBE (-ve) & Mean Ki 67\% PTBE (+ve) & P value \\
56 patients & $1.83 \pm 1.95 \mathrm{SD}$ & $4.83 \pm 4.92 \mathrm{SD}$ & 0.014 \\
\hline
\end{tabular}




\section{Discussion}

Meningiomas are common primary central nervous system (CNS) tumors and account for about $30 \%$ of primary adult intracranial tumors and are usually histologically benign [10] [11] [12]. They are slowly growing tumors that arise from arachnoid cap cells [13]. Meningiomas commonly occur in the fifth and sixth decades of life with a mean age of 48 years [14]. Our study group age showed a wide range, but it is noticed that the incidence proportionately was higher with increasing age, and most commonly patients were in their 5th, 6th and 7th decades, the mean age was around 51 years, which concurs with other previous studies [14] [15] [16]. Meningiomas are more commonly diagnosed in females with a female to male ratio of $2-2.5: 1$ [17] [18] [19] and some authors reported a 3:1 ratio [20]. In our case there was a significant predominance of the female gender as only 8 out of 56 cases encountered were males. Previous reports state that the female predominance in meningiomas may be attributed to hormonal factors and have shown high growth rates of meningiomas during pregnancy and luteal phases of the menstrual cycle [21] [22].

PTBE is commonly found in more than half of meningiomas [11] [12]. Development of PTBE with meningiomas is believed to be contributed to multiple factors, and hasn't been clearly identified, various factors include age, gender, tumor size and location, histopathology and vascularity of the tumor [11] [12] [15] [23] [24] [25]. Multiple studies aimed to identify the causes and mechanisms of PTBE, a study suggested an increased permeability through white matter fibers around the tumor is the cause of PTBE [11], while another study suggested failure of blood brain barrier as the cause of PTBE [26], a widely accepted theory points to the compression of the surrounding brain venous system adjacent to the tumor, leading to increased PTBE [27] [28] [29] [30].

Some studies found a positive correlation between tumor volume and PTBE [30] [31] [32], while other studies found no statistically significant correlation between the tumor volume and PTBE [33] [34] nor with gender [31]. We also found no statistically significant relationship between the tumor volume and PTBE and/or gender, the different results encountered in multiple papers may further enhance the hypothesis that other multiple factors rather than volume that contribute to PTBE development. This explains also why some small meningiomas that are histologically benign can be surrounded by aggressive PTBE causing severe morbidity while other large meningiomas cause no PTBE at all [12] [30].

The prognostic significance of proliferative indices in meningiomas, like the Ki67 index, has been assessed by multiple authors, and it has been suggested that quantifying the tumour's proliferative potential can predict the patient's clinical course and help predict the biological behaviour of individual tumours [35] [36]. High Ki67 index values are associated with increased growth rate and faster doubling time in partially resected meningiomas [24]. A high Ki67 index identifies abnormal proliferation, and therefore broadly speaking, the aggressiveness 
of a tumour [37]. However there is limited data available about the relationship between Ki67 index and certain factors; as peritumoral edema. We aimed in this study to identify if Ki67 index values can be related to the degree of peritumoral edema surrounding meningiomas.

The expression level of Ki67 is known to differ significantly among benign, atypical, and anaplastic meningiomas [38]. Ki67 labeling indices show a highly significant increase from benign (GI) (mean, 3.8\%), through or atypical (GII) (mean, 7.2\%), to anaplastic (GIII) (mean, 14.7\%) meningiomas [39]. Abry et al., reported the average mean labeling indices were $3 \%, 8 \%$, and $17 \%$ for grade I-III meningiomas, respectively [40]. Perry et al., in a study of 62 cases of meningiomas suggested that Ki67 index in excess of $4.2 \%$ was indicative of high tumor proliferation activity and recurrence [41]. Our findings showed a mean value of Ki67 index of 3.28\%, which was near and concurrent with previous literature, no definite cuts off value for Ki67 index could be attributed to inter laboratory variability [36].

In our study, most of Ki67 indices were found to be elevated with PTBE and low in the absence of PTBE, as seen in examples (Figure 4) and (Figure 5) respectively, the mean value of Ki67 index in cases with PTBE was $4.83 \%$ compared to a value of $1.83 \%$ in the cases with absent PTBE (P value 0.014 ), thus a

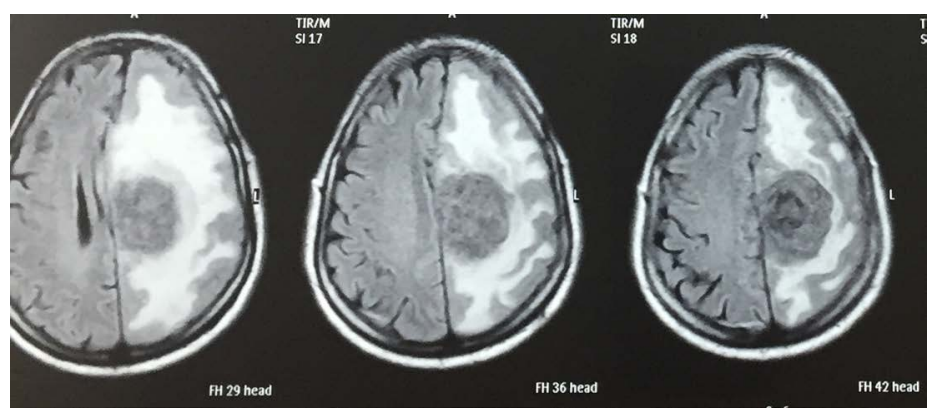

(a)

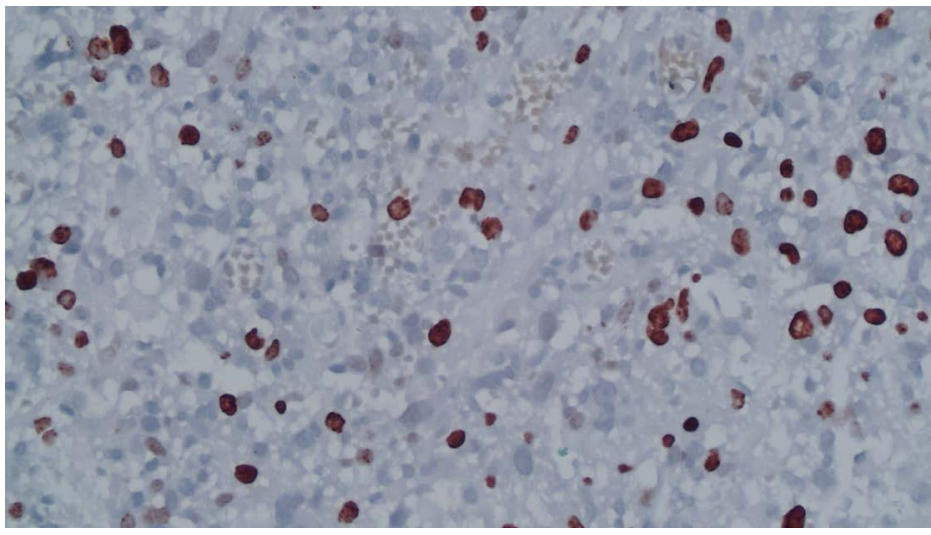

(b)

Figure 4. (a) Axial MRI Flair images of a left falcine meningioma seen as a circumscribed isointense lobulated lesion with significant hyperintense PTBE; (b) Immunohistochemical Ki67 staining of postoperative specimen $(\times 400)$ showed marked nuclear staining demonstrated as dark red/ brown stained nuclei, Ki67 index was 5.2\%. 


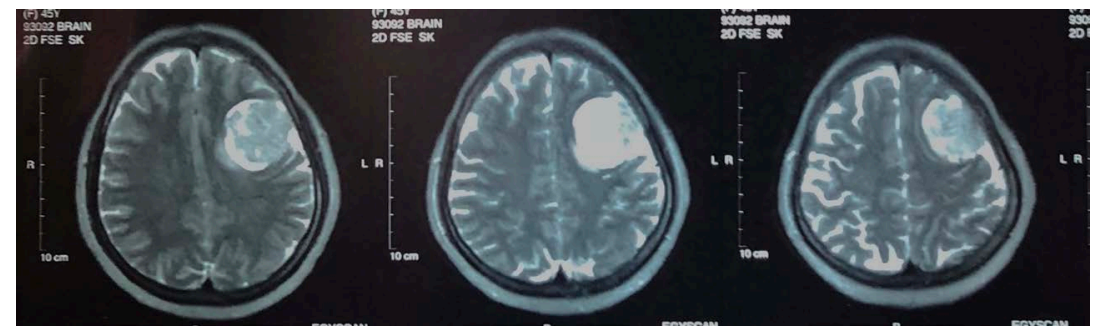

(a)

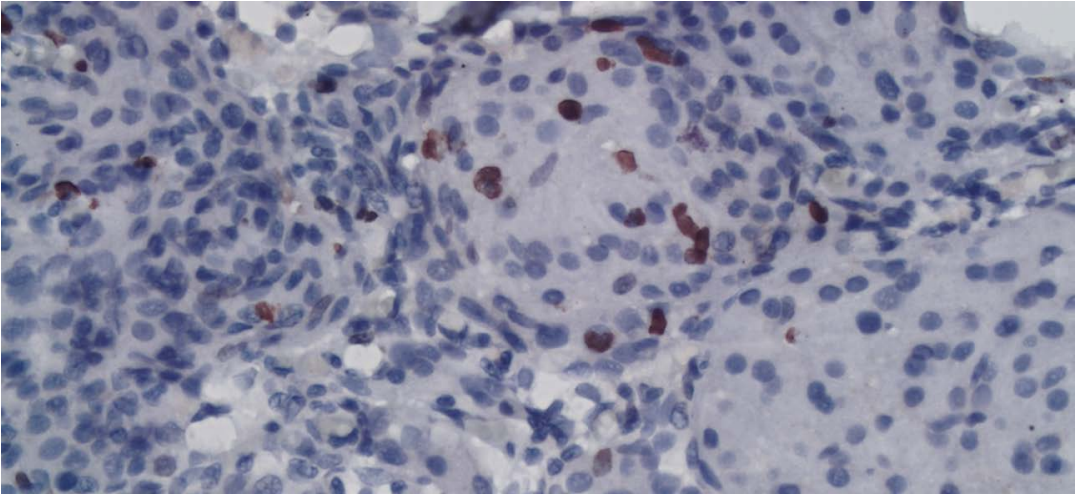

(b)

Figure 5. (a) Axial MRI T2W images of a left convexity meningioma seen as a circumscribed hyperintense lesion without PTBE; (b) Immunohistochemical Ki67 staining of postoperative specimen $(\times 200)$ showed nuclear staining demonstrated as dark red/ brown stained nuclei, Ki67 index was $1.5 \%$.

direct correlation could be proposed between the two parameters, however a study by Gawlitza et al., did not find a relationship between the two parameters [4]. Kim B-W et al., found in their study on 86 patients with meningiomas that higher Ki-67 antigen indices lead to a higher frequency of PTBE [12], which is in agreement with our findings. Other studies also showed a strong correlation between PTBE and Ki67 indices [24] [30] [31] [42] but due to limitations and multiple factors that influence PTBE, further research on larger group of patients and longer follow up periods may be required to conclusively determine the definite relationship.

\section{Conclusion}

In conclusion, we found in our study that peritumoral brain edema surrounding meningiomas may be correlated with their Ki67 index value. High Ki67 indices are evident in meningiomas with surrounding peritumoral brain edema (PTBE). This finding may help shed a better light on the importance of proliferation markers and their role in better prediction of the behavior and characteristics of meningiomas.

\section{Conflicts of Interest}

The authors declare no conflicts of interest regarding the publication of this paper. 


\section{References}

[1] Uduma, U.F. and Emejulu, J.C. (2013) Intracranial Meningiomas in the Present Era of Modern Neuroimaging: Diagnostic and Management Options, with Radiological Illustrations. Orient Journal of Medicine, 25, 67-74.

[2] Harter, P., Braun, Y. and Plate, K. (2017) Classification of Meningiomas: Advances and Controversies. Chinese Clinical Oncology, 6, S2-S2.

https://doi.org/10.21037/cco.2017.05.02

[3] Rockhill, J., Mrugala, M. and Chamberlain, M. (2007) Intracranial Meningiomas: An Overview of Diagnosis and Treatment. Neurosurgical Focus, 23, E1. https://doi.org/10.3171/foc.2007.23.4.2

[4] Gawlitza, M., Fiedler, E., Schob, S., Hoffmann, K. and Surov, A. (2016) Peritumoral Brain Edema in Meningiomas Depends on Aquaporin-4 Expression and Not on Tumor Grade, Tumor Volume, Cell Count, or Ki-67 Labeling Index. Molecular Imaging and Biology, 19, 298-304. https://doi.org/10.1007/s11307-016-1000-7

[5] Whittle, I.R., Smith, C., Navoo, P. and Collie, D. (2004) Meningiomas. The Lancet, 363, 1535-1543. https://doi.org/10.1016/S0140-6736(04)16153-9

[6] Wang, P., Ni, R.Y., Chen, M.N., et al. (2011) Expression of Aquaporin-4 in Human Supratentorial Meningiomas with Peritumoral Brain Edema and Correlation of VEGF with Edema Formation. Genetics and Molecular Research, 10, 2165-2171. https://doi.org/10.4238/vol10-3gmr1212

[7] Ng, W.H., Hy, J.W., Tan, W.L., et al. (2009) Aquaporin-4 Expression Is Increased in Edematous Meningiomas. Journal of Clinical Neuroscience, 16, 441-443. https://doi.org/10.1016/j.jocn.2008.04.028

[8] Tanaka, M., Imhof, H.G., Schucknecht, B., et al. (2006) Correlation between the Efferent Venous Drainage of the Tumor and Peritumoral Edema in Intracranial Meningiomas: Superselective Angiographic Analysis of 25 Cases. Journal of Neurosurgery, 104, 382-388. https://doi.org/10.3171/jns.2006.104.3.382

[9] Reid, M., Bagci, P., Ohike, N., Saka, B., Erbarut Seven, I. and Dursun, N. (2014) Calculation of the Ki67 Index in Pancreatic Neuroendocrine Tumors: A Comparative Analysis of Four Counting Methodologies. Modern Pathology, 28, 686-694. https://doi.org/10.1038/modpathol.2014.156

[10] Challa, S., Babu, S., Uppin, S., Uppin, M., Panigrahi, M., Saradhi, V., Bhattacharjee, S., Sahu, B. and Purohit, A. (2011) Meningiomas: Correlation of Ki67 with Histological Grade. Neurology India, 59, 204.

[11] Bitzer, M., Wöckel, L., Morgalla, M., Keller, C., Friese, S. and Heiss, E. (1997) Peri-tumoural Brain Oedema in Intracranial Meningiomas: Influence of Tumour Size, Location and Histology. Acta Neurochirurgica, 139, 1136-1142. https://doi.org/10.1007/BF01410973

[12] Kim, I.S., Kim, H.D., Kim, K.U., Shin, H.C., Choin, H.J. and Kim, K.H. (1997) Factors Influencing the Development of Peritumoral Brain Edema in Menigiomas. Journal of Korean Neurosurgical Society, 26, 940-945.

[13] Marković, M., Antunović, V., Milenković, S. and Živković, N. (2013) Prognostic Value of Peritumoral Edema and Angiogenesis in Intracranial Meningioma Surgery. Journal of BUON, 18, 430-436.

[14] Sanai, N. and McDermott, M.W. (2010) A Modified Far-Lateral Approach for Large or Giant Meningiomas of the Posterior Fossa. Journal of Neurosurgery, 112, 907-912. https://doi.org/10.3171/2009.6.JNS09120

[15] Gurkanlar, D., Er, U., Sanli, M., Ozkan, M. and Sekerci, Z. (2005) Peritumoral Brain 
Edema in Intracranial Meningiomas. Journal of Clinical Neuroscience, 12, 750-753. https://doi.org/10.1016/j.jocn.2004.09.029

[16] Bhat, A.R., Wani, M.A., Kirmani, A.R. and Ramzan, A.U. (2014) Histological-Subtypes and Anatomical Location Correlated in Meningeal Brain Tumors (Meningiomas). Journal of Neurosciences in Rural Practice, 5, 244-249.

[17] Taghipour, M., Rakei, S.M. and Monabati, A. (2007) The Role of Estrogen and Progesterone Receptors in Grading of the Malignancy of Meningiomas. Iranian Red Crescent Medical Journal, 9, 17-21.

[18] Krayenbuhl, N., Pravdenkova, S. and Al-Mefty, O. (2007) De Novo versus Transformed Atypical and Anaplastic Meningiomas: Comparisons of Clinical Course, Cytogenetics, Cytokinetics, and Outcome. Neurosurgery, 61, 495-503. https://doi.org/10.1227/01.NEU.0000290895.92695.22

[19] Marin Sanabria, E.A., Ehara, K. and Tamaki, N. (2002) Surgical Experience with Skull Base Approaches for Foramen Magnum Meningioma. Neurologia Medico-Chirurgica, 42, 472-480.

[20] Violaris, K., Katsarides, V. and Sakellariou, P. (2012) The Recurrence Rate in Meningiomas: Analysis of Tumor Location, Histological Grading, and Extent of Resection. Open Journal of Modern Neurosurgery, 2, 6-10. https://doi.org/10.4236/ojmn.2012.21002

[21] Lee, E., Grutsch, J., et al. (2006) Association of Meningioma with Reproductive Factors. International Journal of Cancer, 119, 1152-1157.

https://doi.org/10.1002/ijc.21950

[22] Smith, J.S., Quinones-Hinojosa, A., et al. (2005) Sex Steroid and Growth Factor Profile of a Meningioma Associated with Pregnancy. Canadian Journal of Neurological Sciences, 32, 122-127. https://doi.org/10.1017/S0317167100017017

[23] Elster, A.D., Challa, V.R., Gilbert, T.H., Richardson, D.N. and Contento, J.C. (1989) Meningiomas: MR and Histopathologic Features. Radiology, 170, 857-862. https://doi.org/10.1148/radiology.170.3.2916043

[24] Ide, M., Jimbo, M., Yamamoto, M., Umebara, Y., Hagiwara, S. and Kubo, O. (1996) MIB-1 Staining Index and Peritumoral Brain Edema of Meningiomas. Cancer, 78, 133-143.

https://doi.org/10.1002/(SICI)1097-0142(19960701)78:1<133::AID-CNCR19>3.0.C O;2-0

[25] Paek, S.H., Kim, C.Y., Kim, Y.Y., Park, I.A., Kim, M.S., Kim, D.G., et al. (2002) Correlation of Clinical and Biological Parameters with Peritumoral Edema in $\mathrm{Me}$ ningioma. Journal of Neuro-Oncology, 60, 235-245. https://doi.org/10.1023/A:1021186401522

[26] Nam, D.H., Lee, S.K., Whang, S.H., Shin, H.J., Lee, J.I., Kim, J.S., et al. (1998) A Study of the Effects of Clinicobiological Factors upon the Meningioma-Associated Peritumoral Edema Formation. J Korean NeurosurgSoc, 27, 453-459.

[27] Byung-Won, K., Min-Su, K., Sang-Woo, K., Chul-Hoon, C. and Oh-Lyong, K. (2011) Peritumoral Brain Edema in Meningiomas: Correlation of Radiologic and Pathologic Features. Journal of Korean Neurosurgical Society, 49, 26-63. https://doi.org/10.3340/jkns.2011.49.1.26

[28] Nassehi, D. (2013) Intracranial Meningiomas, the VEGF-A Pathway and Peritumoral Brain Edema. Danish Medical Journal, 60, B4626.

[29] Lobato, R.D., Alday, R., Gómez, P.A., Rivas, J.J., Domínguez, J., Cabrera, A., et al. (1996) Brain Oedema in Patients with Intracranial Meningioma. Correlation be- 
tween Clinical, Radiological, and Histological Factors and the Presence and Intensity of Oedema. Acta Neurochirurgica, 138, 485-493.

https://doi.org/10.1007/BF01411166

[30] Tamiya, T., Ono, Y., Matsumoto, K. and Ohmoto, T. (2001) Peritumoral Brain Edema in Intracranial Meningiomas: Effects of Radiological and Histological Factors. Neurosurgery, 49, 1046-1051. https://doi.org/10.1097/00006123-200111000-00003

[31] Bečulić, H., et al. (2019) Correlation of Peritumoral Brain Edema with Morphological Characteristics and Ki67 Proliferative Index in Resected Intracranial Meningiomas. Acta Clinica Croatica, 58, 42-49. https://doi.org/10.20471/acc.2019.58.01.06

[32] Kasuya, H., Kubo, O., Tanaka, M., Amano, K., Kato, K. and Hori, T. (2006) Clinical and Radiological Features Related to Growth Potential of Meningioma. Neurosurgical Review, 29, 293-297. https://doi.org/10.1007/s10143-006-0039-3

[33] Dolecek, T.A., Dressler, E.V., Akkar, J.P., Liu, M., Al-Qaisi, A. and Villano, J.L. (2015) Epidemiology of Meningiomas Post-Public Law 107-206: The Benign Brain Tumor Cancer Registries Amendment Act. Cancer, 121, 2400-2410. https://doi.org/10.1002/cncr.29379

[34] de Azambuja Pereira-Filho, N., Pasqualotto Soares, F., de Mello Chemale, I. and Barbosa Coutinho, L.M. (2010) Peritumoral Brain Edema in Intracranial Meningiomas. Arquivos de Neuro-Psiquiatria, 68, 346-349. https://doi.org/10.1590/S0004-282X2010000300003

[35] Roser, F., Nakamura, M. and Bellinzona, M. (2004) The Prognostic Value of Progesterone Receptor Status in Meningiomas. Journal of Clinical Pathology, 57, 1033-1037. https://doi.org/10.1136/jcp.2004.018333

[36] Mawrin, C. and Perry, A. (2010) Pathological Classification and Molecular Genetics of Meningiomas. Journal of Neuro-Oncology, 99, 379-391. https://doi.org/10.1007/s11060-010-0342-2

[37] Klöppel, G., Couvelard, A., Perren, A., Komminoth, P., McNicol, A. and Nilsson, O. (2008) ENETS Consensus Guidelines for the Standards of Care in Neuroendocrine Tumors: Towards a Standardized Approach to the Diagnosis of Gastroenteropancreatic Neuroendocrine Tumors and Their Prognostic Stratification. Neuroendocrinology, 90, 162-166. https://doi.org/10.1159/000182196

[38] Amatya, V.J., Takeshima, Y. and Sugiyama, K. (2001) Immunohistochemical Study of Ki-67 (MIB-1), p53 Protein, p21WAF1, and p27KIP1 Expression in Benign, Atypical, and Anaplastic Meningiomas. Human Pathology, 32, 970-975.

[39] De Tribolet, N. (2010) Meningiomas: A Comprehensive Text M. Necmettin Pamir, Peter M. Black, Rudolf Fahlbusch. Acta Neurochirurgica, 152, 1445-1445. https://doi.org/10.1007/s00701-010-0622-9

[40] Abry, E., Torp, S.H., Thomassen, I.O. and Salvesen, O.O. (2010) The Significance of Ki-67/MIB-1 Labeling Index in Human Meningiomas: A Literature Study. Pathology-Research and Practice, 206, 810-815. https://doi.org/10.1016/j.prp.2010.09.002

[41] Perry, A., Chicoine, M.R., Filiput, E., Miller, J.P. and Cross, D.T. (2001) Clinicopathologic Assessment and Grading of Embolized Meningiomas: A Correlative Study of 62 Patients. Cancer, 92, 701-711. https://doi.org/10.1002/1097-0142(20010801)92:3<701::AID-CNCR1373>3.0.CO;2-7

[42] Aguiar, P.H., Tsanaclis, A.M. and Tella Jr., O.I. and Plese, J.P. (2003) Proliferation Rate of Intracranial Meningiomas as Defined by the Monoclonal Antibody MIB-1. Neurosurgical Review, 26, 221-228. https://doi.org/10.1007/s10143-003-0261-1 